\section{Mortalidade e causas de óbitos nas prisões do Rio de Janeiro, Brasil}

\author{
Mortality and causes of deaths in prisons in \\ Rio de Janeiro, Brazil
}
Mortalidad y causas de óbitos en las prisiones de Río de Janeiro, Brasil

\author{
Rio de Janeiro, Brasil
}

Alexandra Sánchez 1

Celina Roma Sánchez de Toledo 1

Luiz Antônio Bastos Camacho 1

Bernard Larouze 1

\title{
Resumo
}

A mortalidade nas prisões, indicador fundamental do direito à saúde das pessoas privadas de liberdade (PPL), nunca foi estudada de maneira aprofundada no Brasil. A avaliação da mortalidade global e por causas entre PPL encarceradas em 2016-2017 no Estado do Rio de Janeiro, foi realizada a partir de dados do Sistema de Informações sobre Mortalidade e da Administração Penitenciária. Taxas de mortalidade entre PPL e população geral do estado foram comparadas após padronização. As principais causas de morte entre PPL foram doenças infecciosas (30\%), doenças do aparelho circulatório (22\%) e causas externas (12\%). Dentre as causas infecciosas, destacam-se HIV/aids (43\%) e tuberculose (TB) (52\% se considerados todos os óbitos com menção de TB). Somente 0,7\% das PPL que faleceram tiveram acesso a serviço de saúde extramuros. A taxa global de mortalidade foi maior entre as PPL comparadas à população geral do estado, com mortalidade por doenças infecciosas 5 vezes superior, por TB 15 vezes e por doenças endócrinas, especialmente diabetes, e doenças circulatórias (1,5 e 1,3 vez, respectivamente), enquanto mortes por causa externa foram menos frequentes entre PPL. Este estudo mostra um expressivo excesso de mortes potencialmente evitáveis nas prisões, o que traduz importante desassistência e exclusão dessa população do Sistema Único de Saúde. Evidencia a necessidade de um sistema de monitoramento, em tempo real, dos óbitos, preciso e sustentável, além da reestruturação da saúde prisional por meio da efetivação da Política Nacional de Atenção Integral à Saúde das Pessoas Privadas de Liberdade no Sistema Prisional para que as PPL usufruam do direito constitucional à saúde em sua integralidade, com a mesma qualidade e tempestividade oferecida à população geral.

Prisões; Mortalidade; Causa do Óbito; Tuberculose; Vigilância Epidemiológica

\author{
Correspondência \\ A. Sánchez \\ Escola Nacional de Saúde Pública Sergio Arouca, Fundação \\ Oswaldo Cruz. \\ Rua Leopoldo Bulhões 1480, 6o andar, Rio de Janeiro, RJ \\ 21041-210, Brasil. \\ asanchez.fiocruz@gmail.com \\ 1 Escola Nacional de Saúde Pública Sergio Arouca, Fundação \\ Oswaldo Cruz, Rio de Janeiro, Brasil.
}




\section{Introdução}

A mortalidade nas prisões brasileiras geralmente é conhecida pelas informações difundidas pela mídia e sociedade civil ou compiladas pelo Departamento Penitenciário Nacional (Ministério da Justiça) a partir de dados fornecidos pelos estados da Federação 1. Estas informações apresentam importantes limitações: são dados agregados informados semestralmente a partir de informações coletadas em formulário padronizado, cuja precisão não é assegurada, e as causas dos óbitos são classificadas simplesmente como natural, criminal, suicídio, acidental ou indeterminada, e não de acordo com a 10a Revisão da Classificação Internacional de Doenças (CID-10) da Organização Mundial da Saúde (OMS). A fragilidade e a inconsistência desses dados dificultam sua utilização como indicador para medir a qualidade da assistência e para subsidiar a elaboração e/ou reorientação das políticas de saúde prisional. Por outro lado, a partir do Sistema de Informações sobre Mortalidade (SIM), não é possível identificar a origem prisional dos óbitos, o que impossibilita, na rotina, a utilização dessas informações.

Nas prisões brasileiras, cerca de 780 mil pessoas estão privadas de liberdade (PPL) em celas coletivas, mal ventiladas (até $170 \mathrm{PPL} /$ cela), superlotadas (média de 172\%, com variação de $108 \%$ a $267 \%$ entre os estados), com precárias condições de higiene - agravadas, inclusive, em alguns estados, pelo acesso limitado à água. Essas condições de encarceramento, aliadas às deficiências do sistema de saúde prisional 2, são responsáveis por expressiva morbidade 3 , especialmente, por doenças infecciosas como a tuberculose (TB) 4,5 e o HIV/aids 6,7.

A atenção à saúde nas prisões, regulamentada pela Portaria Interministerial MJ/MS no 1/2014 que estabelece a Política Nacional de Atenção Integral à Saúde das Pessoas Privadas de Liberdade no Sistema Prisional (PNAISP) 8, visa garantir o acesso das PPL ao cuidado integral no Sistema Único de Saúde (SUS). No entanto, o grau de implementação dessa política, que tem como princípios a integralidade da atenção à saúde, a intersetorialidade (parceria saúde/justiça) e a territorialidade, com articulação da atenção básica às redes de atenção especializada, hospitalar e de urgência/emergência extramuros, varia nos diferentes estados da Federação. No Estado do Rio de Janeiro, até o momento da realização deste estudo, sua implantação era muito incipiente, sendo a administração penitenciária responsável pela atenção à saúde das PPL.

$\mathrm{Na}$ literatura científica, estudos sobre a mortalidade nas prisões são, em sua maioria, antigos e realizados em países de alta renda 9,10,11,12, onde as condições de encarceramento, especialmente no que se refere à superlotação e à assistência à saúde são melhores do que no Brasil. Nesse contexto, estudos aprofundados sobre mortalidade nas prisões brasileiras são fundamentais para estimar o estado de saúde dessa população, a qualidade das ações de prevenção e de assistência dispensadas e fornecer elementos que permitam desenvolver ou adaptar estratégias de saúde preventiva e curativa, além de avaliar se o direito à saúde e à vida das PPL, que é um dever do Estado 13, é efetivamente respeitado.

Assim, o estudo que realizamos nas prisões do Estado do Rio de Janeiro teve por objetivo avaliar as taxas de mortalidade e causas dos óbitos entre as PPL, nos anos de 2016 e 2017, e compará-las às da população geral do estado, a fim de contribuir para a adequação das estruturas e do funcionamento do sistema de saúde prisional e sua articulação com serviços de saúde extramuros. Contribuíram para a escolha do local de estudo e o recorte temporal, as denúncias feitas pela Defensoria Pública do Rio de Janeiro 14 e pela ONG Instituto Igarapé sobre a ocorrência de grande número de óbitos de 2015 a 2017 nas prisões do estado, sem que as causas fossem conhecidas.

\section{População prisional do Estado do Rio de Janeiro e a estrutura de saúde intramuros}

Em meados de 2017, cerca de 50 mil pessoas estavam encarceradas nas 45 unidades prisionais (UP) do estado, das quais, 4,5\% eram mulheres. Os presos provisórios representavam 30,6\% da população composta por adultos jovens $(93,1 \%$ com idade $<45$ anos), em sua maioria pretos ou pardos $(73,3 \%) 1$. Mais da metade das PPL (54,4\%) estavam custodiadas em UPs localizadas no Complexo Penitenciário de Gericinó (Município do Rio de Janeiro); 37,7\%, em UPs isoladas do Município do Rio de Janeiro e da Região Metropolitana; e 7,9\%, em UPs do interior do estado. A taxa de ocupação média das UPs era de $177 \%$ (variação: 140 a $300 \%$ conforme a UP).

O sistema de saúde prisional, sob a gestão da Secretaria de Estado de Administração Penitenciária (SEAP-RJ), contava com um ambulatório de saúde em cada UP, com equipe técnica limitada a técnicos 
de enfermagem, e, no Complexo de Gericinó, dois hospitais psiquiátricos, uma unidade de referência para diagnóstico e tratamento de TB (Sanatório Penal) e, sob a gestão da Secretaria de Estado de Saúde (SES-RJ), uma Unidade de Pronto Atendimento Prisional (UPA). O Sanatório Penal dotado de recursos para diagnóstico da TB, além da hospitalização de casos de maior complexidade e isolamento de casos de resistência ou multirresistência, realiza atendimento ambulatorial para investigação diagnóstica e acompanhamento do tratamento, avaliação da alta e supervisão do tratamento nas UPs. Devido à redução progressiva do número de médicos e enfermeiras nas UPs e no próprio Sanatório Penal, a UPA prisional passou a centralizar a maior parte das demandas de saúde, inclusive os casos presumidos de TB, embora sem estrutura para diagnóstico bacteriológico. Os pacientes portadores de transtornos mentais, desde que estáveis, eram mantidos nas UPs, onde o acompanhamento médico especializado e acesso a medicamentos eram erráticos.

Por obrigação legal 15, os corpos de todas PPL que falecem, seja por causa natural ou externa, devem ser encaminhados ao Instituto Médico Legal (IML) para identificação legal, realização de necrópsia e emissão da Declaração de Óbito (DO), que servirá para alimentar o SIM.

\section{Método}

Foram incluídas, no estudo, todas as PPL que estavam encarceradas entre janeiro de 2016 e dezembro de 2017 nas UPs do Estado do Rio de Janeiro, e, considerados todos os óbitos ocorridos nas UPs ou em serviços de saúde intramuros (do próprio sistema prisional) ou extramuros (da rede de serviços para a população livre).

Informações sobre o número de PPL e a lista nominal dos óbitos ocorridos no período de interesse foram obtidas nos registros da SEAP-RJ. Esses foram relacionados com a base de dados do SIM no Rio de Janeiro (SIM/RJ) por meio do aplicativo RecLink II, versão 3.1 (http://reclink.sourceforge. net/), utilizando as seguintes variáveis: nome, nome da mãe, data do nascimento e data do óbito. Causas básicas do óbito obtidas a partir do SIM/RJ, com consulta à DO, foram codificadas de acordo com a CID-10. Para todos os óbitos com causa indeterminada e aqueles por doenças infecciosas e parasitárias, doenças do aparelho circulatório e do aparelho respiratório na DO, agravos que concentravam a maior parte dos óbitos, dados complementares foram buscados em boletins de atendimento médico e prontuários de saúde, e, quando pertinente, a causa básica foi recodificada de acordo com a CID-10.

As taxas de mortalidade na população geral foram calculadas a partir de dados do Instituto Brasileiro de Geografia e Estatística (IBGE) e do SIM/RJ. Para o cálculo das taxas de mortalidade geral e por grupos de causas, foi utilizado, como denominador, a soma da população que se encontrava presa em junho de cada um dos dois anos. Também foi calculada a mortalidade proporcional por grandes grupos de causa (causa externa e natural) e, entre as causas naturais, a mortalidade por capítulos da CID-10.

A partir do efetivo carcerário total e do número de óbitos, foram calculados o risco relativo (RR) de morte e seu respectivo intervalo de 95\% de confiança (IC95\%), associado a sexo, localização geográfica da UP, situação jurídica (presos provisórios/condenados) e taxa de ocupação da UP.

Para comparar a mortalidade na população prisional com a da população geral do Rio de Janeiro, ajustando para as diferenças nas distribuições etárias, a razão de mortalidade padronizada (standardized mortality ratio, SMR) 16 foi calculada pelo quociente do número observado de óbitos (total e por grupos de causas) nas prisões do Estado do Rio de Janeiro e a soma dos óbitos estimados por faixa etária da população prisional, aplicando as taxas de mortalidade específicas correspondentes na população do Rio de Janeiro (http://sistemas.saude.rj.gov.br/tabnet/deftohtm.exe?sim/obito.def, acessado em 06/Set/2019), após exclusão de acidentes de transporte. Dado o baixo número de óbitos entre mulheres presas, essas análises foram limitadas à população masculina. A padronização por idade considerou homens com idades entre 18 e 89 anos. As faixas etárias foram definidas a partir das categorias utilizadas pela Organização Pan-Americana da Saúde (OPAS), adaptadas segundo as caraterísticas da população estudada, constituída, majoritariamente, por jovens com 18 anos ou mais. Para a análise dos dados, foi utilizado o software SPSS for Windows, versão 20.0 (https://www.ibm. com/), e o software WINPEPI, versão 11.65 (http://www.brixtonhealth.com/pepi4windows.html), foi utilizado para estimar os limites de $95 \%$ de confiança dos SMR 17. 
O protocolo do estudo foi aprovado pelo Comitê de Ética em Pesquisa da Escola Nacional de Saúde Pública Sergio Arouca, Fundação Oswaldo Cruz (parecer no 2.600.000, de 16 de abril de 2018).

\section{Resultados}

Dos 540 óbitos informados pela SEAP-RJ (266 em 2016 e 274 em 2017), 22 (4,1\%) não foram localizados no SIM, e, dentre os 518 localizados, em 13 casos (2,5\%), a DO não foi encontrada. Dos 22 óbitos não localizados no SIM (8 em 2016 e 14 em 2017), 16 eram de UPs localizadas fora do Complexo de Gericinó, 21 eram homens, 11 óbitos ocorreram na UPA prisional, 8 na UP, e, em 3, o local do óbito não foi informado. Assim, a análise da causa do óbito só foi possível em 93,5\% (505/540) dos óbitos informados. Em 35 (6,9\%) dos 505 óbitos com DO, o nome do falecido não constava no documento. Entretanto, por meio de busca no SIM, pela data aproximada do óbito, sexo e do endereço do local de ocorrência do óbito, foi possível a identificação presumida de 31 (88,6\%) desses falecidos.

A revisão de documentos adicionais, realizada em 62,2\% dos óbitos (314/505), permitiu, a partir da história clínica e de exames laboratoriais encontrados em prontuários médicos, a identificação das causas de óbitos que figuravam como indeterminadas ou a retificação da causa básica em 47,5\% dos óbitos revisados, o que corresponde a $29,5 \%$ do total dos óbitos. A título de exemplo, permitiu a identificação de 23 óbitos por causas metabólicas, 21 deles por diabetes mellitus, não citadas na DO antes dessa revisão, assim como óbitos inicialmente considerados como por doenças respiratórias que foram recodificados como TB. Permitiu ainda a evidenciação de transtorno mental como causa associada a óbitos por agressão durante surtos, além de epilepsia com broncoaspiração durante crise convulsiva como determinante da morte. A necrópsia tinha sido realizada em $23,8 \%$ do total de óbitos e em $38,2 \%$ das mortes por causa externa.

A taxa global de mortalidade observada foi de 53,29/10 mil e 53,78/10 mil, em 2016 e 2017, respectivamente (mulheres: 22.6 e 33.1/10 mil e homens: 54,71 e 54,684/10 mil, em 2016 e 2017). Para as análises posteriores, foram consideradas as informações dos dois anos agregadas.

A Tabela 1 mostra que os óbitos eram predominantemente de homens, jovens, pretos ou pardos. Como mostrado na Tabela 2, a mortalidade foi superior entre homens custodiados em UPs do interior do estado ou em unidades isoladas no Município do Rio de Janeiro e na Região Metropolitana, em relação àquelas localizadas no Complexo de Gericinó. A taxa de mortalidade também foi superior entre presos provisórios em relação aos condenados. Na falta de informação sobre a idade das pessoas presas que não faleceram, não foi possível estimar o risco relativo por idade. Não foi evidenciada associação estatisticamente significativa entre o óbito e a superlotação da UP.

A análise da distribuição proporcional segundo a causa básica dos óbitos mostra que as causas naturais foram as mais frequentes, responsáveis por $83,7 \%$ dos óbitos $v s .11,9 \%$ por causas externas (Tabela 3). Entre as causas naturais, as doenças infecciosas e parasitárias foram as mais frequentes, responsáveis por $28,5 \%$ do total dos óbitos, concentrados em três principais agravos, que correspondem a 92\% (138/150) dessas mortes: TB, responsável por 40,7\% $(\mathrm{n}=61)$, HIV/aids 42,7\% $(\mathrm{n}=64)$ e septicemia $13,3 \%(\mathrm{n}=20)$. A TB estava implicada em 26,6\% (17/64) dos óbitos por HIV/aids, o que eleva para $52 \%$ os óbitos com menção de TB. Entre os 61 óbitos por TB sem infecção pelo HIV/aids, 19,7\% (12/61) morreram em estado de caquexia; 11,5\% (7/61), por hemoptise; e 6,6\% (4/61) eram diabéticos. Em 65,5\% dos casos, a TB foi diagnosticada durante a internação em que ocorreu o óbito.

Entre os 64 óbitos pelo HIV/aids, 97\% foram atribuídos a infecções, tais como: TB (17 óbitos), pneumonias (7), das quais 4 por Pneumocysti carinii, infecções do sistema nervoso central (5), doenças múltiplas (3) e infecções não especificadas (25). Dois óbitos foram atribuídos a neoplasias, e 5 óbitos, sem informação sobre a causa. Em relação às septicemias, terceira causa de óbito por doenças infecciosas, pneumonia e infecção de pele estiveram implicadas em grande parte dos óbitos para os quais obtivemos informação sobre a origem da infecção.

Doenças do aparelho circulatório contribuíram com $22 \%$ dos óbitos, sendo os agravos mais frequentes o infarto agudo do miocárdio (36 óbitos), cardiomiopatias (28), insuficiência cardíaca (14), embolia pulmonar (12), transtornos vasculares cerebrais (7) e aneurismas (4). Quanto aos 55 óbitos classificados como doenças respiratórias, responsáveis por 10,4\% do total, predominou a pneumonia 


\section{Tabela 1}

Características descritivas das pessoas privadas de liberdade (PPL) que evoluíram para óbito no Estado do Rio de Janeiro, Brasil, 2016-2017.

\begin{tabular}{|c|c|c|}
\hline Características & $\mathbf{n}$ & $\%$ \\
\hline \multicolumn{3}{|l|}{ Faixa etária (anos) } \\
\hline $18-35$ & 251 & 50,2 \\
\hline $36-59$ & 182 & 36,4 \\
\hline$>60 *$ & 67 & 13,4 \\
\hline \multicolumn{3}{|l|}{ Raça/Cor } \\
\hline Branca & 132 & 26,5 \\
\hline Preta & 91 & 18,2 \\
\hline Parda & 276 & 55,3 \\
\hline \multicolumn{3}{|l|}{ Sexo } \\
\hline Masculino & 528 & 97,8 \\
\hline Feminino & 12 & 2,2 \\
\hline \multicolumn{3}{|l|}{ Alocadas em UPs localizadas } \\
\hline Fora do Complexo de Gericinó & 281 & 53,9 \\
\hline No Complexo de Gericinó & 240 & 46,1 \\
\hline \multicolumn{3}{|l|}{ Situação jurídica } \\
\hline Presos condenados & 203 & 39,0 \\
\hline Presos provisórios & 174 & 33,4 \\
\hline Desconhecida ** & 144 & 27,6 \\
\hline \multicolumn{3}{|c|}{ Alocadas em UPs com taxa de ocupação (\%) } \\
\hline$<140$ & 105 & 20,3 \\
\hline$>140$ a 199 & 179 & 34,7 \\
\hline$>199$ & 232 & 45,0 \\
\hline
\end{tabular}

UP: unidade prisional.

Fonte: Secretaira de Estado de Administração Penitenciária do Rio de Janeiro e Ministério Público do Estado do Rio de Janeiro, 2016 e 2017.

* Incluindo 6 PPL entre 81-87 anos;

** Refere-se a presos cuja situação jurídica não pode ser identificada a partir das informações disponíveis, por estarem alocados em prisões que abrigavam tanto presos condenados quanto provisórios.

(25 óbitos), seguida por abscesso pulmonar (5) e asma (3). Nesse grupo, em 16 óbitos, não foi possível determinar a doença respiratória responsável pela morte.

A diabetes mellitus, inicialmente não figurava no SIM como causa básica ou associada em nenhum óbito na população estudada. Após a revisão de prontuários, foram identificados 33 óbitos com menção da diabetes, o que corresponde a 6\% do total de óbitos, sendo mencionada em 12 óbitos, como doença associada e, em 21 óbitos, como causa básica (91\% das doenças do Capítulo IV da CID-10). As principais condições associadas foram infecções em 16 óbitos (pneumonia, infecção urinária e de pele), hipoglicemia (4 óbitos) e doenças cardíacas (4 óbitos).

Entre as causas externas, responsáveis por 63 óbitos (11,9\% do total de óbitos), houve predomínio da violência (54\%), suicídio (11,1\%), e 34,9\% foram classificadas como "acidente/intenção indeterminada", sem que nossa busca por informações adicionais permitisse melhor esclarecer a real causa desses óbitos. Vale ressaltar a identificação de, pelo menos, três mortes por agressão a pacientes com distúrbios mentais durante crises. Considerando o baixo número (11 óbitos: 4 em 2016 e 7 em 2017) e a heterogeneidade das causas de óbito entre as mulheres, sem nenhum óbito relacionado à gestação ou ao parto, a comparação com a população geral foi limitada à população masculina.

A comparação da mortalidade entre homens presos e na população geral masculina do Estado do Rio de Janeiro, após padronização por idade (Tabela 4), mostrou taxas globais superiores entre as PPL, 
Tabela 2

Risco relativo de morte entre pessoas privadas de liberdade (PPL) no Estado do Rio de Janeiro, Brasil, segundo o sexo e características do encarceramento, 2016-2017.

\begin{tabular}{|c|c|c|c|}
\hline Variável & Óbitos (n) & PPL (n) & RR (IC95\%) \\
\hline \multicolumn{4}{|l|}{ Sexo } \\
\hline Feminino & 12 & 4.232 & 1,00 \\
\hline Masculino & 528 & 97.046 & $1,92(1,08-3,40$ \\
\hline \multicolumn{4}{|l|}{ Localização da UP } \\
\hline Complexo de Gericinó & 240 & 60.054 & 1,00 \\
\hline Fora do Complexo de Gericinó & 281 & 40.387 & $1,74(1,47-2,07)$ \\
\hline \multicolumn{4}{|l|}{ Situação jurídica } \\
\hline Preso condenado & 203 & 48.256 & 1,00 \\
\hline Preso provisório & 174 & 29.440 & $1,40(1,15-1,72)$ \\
\hline Preso em UP mista & 144 & 23.582 & $1,45(1,17-1,80)$ \\
\hline \multicolumn{4}{|l|}{ Taxa de ocupação (\%) } \\
\hline$<140$ & 105 & 19.390 & 1,00 \\
\hline > 140 a 199 & 179 & 31.507 & $1,05(0,83-1,33)$ \\
\hline$>199$ & 232 & 49.898 & $0,86(0,70-1,10)$ \\
\hline
\end{tabular}

IC95\%: intervalo de 95\% de confiança; RR: risco relativo; UP: unidade prisional.

Fonte: Secretaira de Estado de Administração Penitenciária do Rio de Janeiro e Ministério Público do Estado do Rio de Janeiro, 2016 e 2017.

Nota: UP ignorada em 19/540 óbitos.

com SMR = 1,30 (IC95\%: 1,15-1,45). A mortalidade por causas externas foi 2 vezes mais elevada fora da prisão ( $\mathrm{SMR}=0,31$, IC95\%: 0,24-0,40), enquanto a mortalidade por doenças infecciosas e parasitárias foi 5 vezes superior entre as PPL (SMR =6,04; IC95\%: 5,11-7,06). Entre as doenças infecciosas, os óbitos por TB foram 15 vezes superiores (SMR = 16,21; IC95\%: 12,41-20,84) na população encarcerada, assim como HIV/aids 3 vezes (SMR = 4,06; IC95\%: 2,96-5,43) e, por coinfecção TB-HIV, mais de 5 vezes $(\mathrm{SMR}=6,53$; IC95\%: 3,73-10,60) superiores.

A maioria dos óbitos (93,8\%) ocorreu intramuros, sendo 63,1\% (342 óbitos) na UPA, 29\% (157 óbitos) na própria unidade prisional e 1,9\% (9) no hospital para TB. Quatro PPL morreram em hospitais públicos fora do sistema prisional (0,7\%), e, em 30 (5,5\%), não foi possível identificar o local do óbito.

\section{Discussão}

Os resultados deste estudo de caso nas prisões do Estado do Rio de Janeiro mostraram taxa de mortalidade global maior do que a da população geral, com mortalidade por doenças infecciosas, em sua maioria curáveis, muito superior na população privada de liberdade. Por outro lado, as mortes por causa externa foram mais frequentes na população geral.

Trata-se, a nosso ver, do primeiro estudo aprofundado sobre óbitos e suas causas entre as PPL no Brasil e, de maneira geral, nos países de média e baixa renda. Por implicar na responsabilidade estatal em assegurar a saúde e a vida das pessoas presas 18, a mortalidade e todas as informações relativas à péssima situação das prisões são temas sensíveis para administração penitenciária, que se esforça em manter essas questões fora do debate público, dificultando o acesso à informação detalhada e individualizada 19,20. 
Tabela 3

Mortalidade proporcional segundo a causa básica por capítulos da 10a Revisão da Classificação Internacional de Doenças (CID-10) em pessoas privadas de liberdade (PPL) no sistema penitenciário do Estado do Rio de Janeiro, Brasil, 2016-2017.

\begin{tabular}{|c|c|c|c|c|}
\hline \multirow[t]{3}{*}{ Capítulos da CID-10 } & \multicolumn{4}{|c|}{ Óbitos } \\
\hline & \multicolumn{2}{|c|}{ Sistema penitenciário } & \multicolumn{2}{|c|}{ População geral } \\
\hline & $\mathbf{n}$ & $\%$ & $\mathbf{n}$ & $\%$ \\
\hline I - Algumas doenças infecciosas e parasitárias & 150 & 28,5 & 7.845 & 5,9 \\
\hline II - Neoplasmas & 18 & 3,4 & 21.334 & 16,0 \\
\hline III - Doenças do sangue e dos órgãos hematopoiéticos e alguns transtornos imunitários & 1 & 0,2 & 719 & 0,5 \\
\hline IV - Doenças endócrinas, nutricionais e metabólicas & 23 & 4,4 & 7.110 & 5,3 \\
\hline V-Transtornos mentais e comportamentais & 1 & 0,2 & 1.089 & 0,8 \\
\hline $\mathrm{VI}$ - Doenças do sistema nervoso & 11 & 2,1 & 2.768 & 2,1 \\
\hline IX - Doenças do aparelho circulatório & 116 & 22,0 & 40.296 & 30,3 \\
\hline X - Doenças do aparelho respiratório & 55 & 10,4 & 16.053 & 12,1 \\
\hline$X I$ - Doenças do aparelho digestivo & 31 & 5,9 & 6.475 & 4,9 \\
\hline XII - Doenças da pele e do tecido subcutâneo & 2 & 0,4 & 749 & 0,6 \\
\hline $\begin{array}{l}\text { XVIII - Sintomas, sinais e achados anormais de exames clínicos e de laboratório, não } \\
\text { classificados em outro capítulo }\end{array}$ & 26 & 4,9 & 5.201 & 3,9 \\
\hline XX - Causas externas de morbidade e de mortalidade * & 63 & 11,9 & 8.801 & 6,6 \\
\hline Sem informação & 22 & 4,2 & & \\
\hline Total & 527 & 100,0 & 133.079 & 100,0 \\
\hline
\end{tabular}

* Excluído acidente de transporte.

Fonte: Ministério da Saúde (Sistema de Informaç̃̃es sobre Mortalidade e Departamento de Informática do SUS), 2016 e 2017.

\section{Tabela 4}

Razão de mortalidade padronizada por idade na população privada de liberdade (PPL) no sistema penitenciário do Estado do Rio de Janeiro, tendo, como referência, a população geral do Estado do Rio de Janeiro, Brasil, 2016-2017.

\begin{tabular}{|c|c|c|c|c|}
\hline \multirow[t]{2}{*}{ Capítulo da CID-10 } & \multicolumn{2}{|c|}{ Óbitos } & \multirow[t]{2}{*}{ SMR * } & \multirow[t]{2}{*}{ IC95\% } \\
\hline & Observados & Esperados & & \\
\hline I- Algumas doenças infecciosas e parasitárias & 147 & 24,31 & 6,045 & $5,11-7,07$ \\
\hline II - Neoplasmas & 17 & 26,29 & 0,646 & $0,38-10,35$ \\
\hline III - Doenças do sangue e dos órgãos hematopoiéticos e alguns transtornos imunitários & 1 & 1,79 & 0,558 & $0,01-3,09$ \\
\hline IV - Doenças endócrinas, nutricionais e metabólicas & 22 & 8,87 & 2,479 & $1,55-3,76$ \\
\hline V-Transtornos mentais e comportamentais & 1 & 3,34 & 0,299 & $0,01-1,67$ \\
\hline VI - Doenças do sistema nervoso & 11 & 5,35 & 2,055 & $1,02-3,68$ \\
\hline IX - Doenças do aparelho circulatório & 114 & 50,60 & 2,253 & $1,75-2,83$ \\
\hline X-Doenças do aparelho respiratório & 56 & 18,83 & 2,973 & $2,25-3,87$ \\
\hline XI - Doenças do aparelho digestivo & 30 & 10,97 & 2,734 & $1,85-3,90$ \\
\hline XII - Doenças da pele e do tecido subcutâneo & 2 & 0,92 & 2,172 & $0,26-7,85$ \\
\hline XIV - Doenças do aparelho geniturinário & 8 & 5,12 & 1,560 & $0,67-3,07$ \\
\hline $\begin{array}{l}\text { XVIII - Sintomas, sinais e achados anormais de exames clínicos e de laboratório, não } \\
\text { classificados em outro capítulo }\end{array}$ & 23 & 23,17 & 0,992 & $0,62-1,49$ \\
\hline$X X$ - Causas externas de morbidade e de mortalidade ** & 61 & 192,85 & 0,316 & $0,24-0,40$ \\
\hline Total & 494 & 379,54 & 1,302 & $1,16-1,46$ \\
\hline
\end{tabular}

CID-10: $10^{a}$ Revisão da Classificação Internacional de Doenças; IC95\%: intervalo de 95\% de confiança; SMR: razão de mortalidade padronizada. Fonte: Ministério da Saúde (Sistema de Informações sobre Mortalidade e Departamento de Informática do SUS), 2016 e 2017.

* Padronização por idade (homens entre 18 e 89 anos) pelo método indireto;

** Excluído acidente de transporte. 
Assim, nosso estudo, com retorno aos dados primários para estabelecer a realidade dos fatos, aponta o alto grau de imprecisão em relação à causa mortis evidenciado pelo elevado percentual de reclassificação da causa básica após revisão documental. Nesse sentido, ressalta-se, dentre outras, a necessidade da realização sistemática da necrópsia de todas as PPL falecidas, como previsto em lei no Brasil, e que o presente estudo mostrou ter sido realizada em menos de 1/3 dos óbitos no Rio de Janeiro.

A menor mortalidade observada nas unidades prisionais do Complexo de Gericinó, que abrigam cerca da metade das PPL do estado, pode ser explicada pela centralização, nesse Complexo, dos principais serviços de saúde prisional (UPA e Sanatório Penal). Isso mostra a importância da integração de serviços de atenção básica das UPs com a rede de serviços de saúde extramuros nas cidades onde as prisões estão localizadas, como preconizado no Brasil pela PNAISP 8 . Entretanto, essa descentralização tarda a se concretizar, em parte, pela carga adicional de trabalho que significará para os municípios implicados e pela complexidade dos mecanismos administrativos envolvidos.

A constatação, em nosso estudo, de maior mortalidade entre presos provisórios, que podem permanecer encarcerados, não raro por meses ou anos enquanto aguardam julgamento, quando comparada aos presos condenados não tem explicação clara. No Rio de Janeiro, as condições ambientais e de assistência à saúde são semelhantes entre as prisões para provisórios e condenados, e não observamos diferenças significativas em relação à frequência relativa das diferentes causas de morte, nem às características sociodemográficas. Não se pode, entretanto, afastar a possibilidade de que os condenados sejam "sobreviventes", isto é, que os provisórios com maior risco de morrer sejam retirados prematuramente do grupo dos condenados. Essa maior mortalidade de presos provisórios pode também estar associada a um conjunto de fatores que incluem a inexistência de exame admissional, a descontinuidade do tratamento de doenças existentes por ocasião do encarceramento, as dificuldades iniciais de adaptação desses presos ao universo carcerário e o seu complexo funcionamento, altamente hierarquizado, que tem o acesso à saúde regulado não só pelos guardas, mas também pelos próprios PPL 19,21. A ausência, em nosso estudo, de relação entre a mortalidade e a taxa de ocupação (superlotação) pode estar relacionada às altas taxas de ocupação verificadas em todas as UPs.

As mortes por doenças infecciosas, especialmente por TB, HIV/aids e septicemias que correspondem a quase $1 / 3$ dos óbitos e determinam risco de morrer 5 vezes superior ao da população geral do estado (SMR = 6,04; IC95\%: 5,11-7,06), contrastam com dados de estudos em países de alta renda onde, mesmo após o aparecimento do HIV/aids nos anos 1980, predominam as mortes por doenças crônicas, especialmente as cardiovasculares 9,11,22.

A predominância da TB entre as causas de óbito está em coerência com a grave situação epidemiológica observada nas prisões do Rio de Janeiro 4, estado que apresenta as maiores taxas de incidência do país 23 entre PPL e onde inquéritos de busca ativa por rastreamento radiológico têm mostrado, ao longo dos anos, elevadas taxas de prevalência de TB (5\% a 10\%) ${ }^{4}$. Esse excesso de mortes por TB nas prisões, 15 vezes superior em relação à população geral (SMR = 16,20; IC95\%: 12,41-20,84), ocorre em um contexto de baixa taxa de resistência às drogas, de coinfecção pelo HIV de cerca de $12 \%$ e acesso regular ao tratamento supervisionado para os casos detectados. Entretanto, medidas de controle limitadas à detecção de casos a partir da demanda espontânea, sem a realização, como rotina, de busca ativa sistemática, deixam de detectar muitos casos, e a baixa resolutividade dos serviços intramuros são responsáveis pela alta mortalidade observada. O elevado percentual de mortes por TB em estado de caquexia e o fato de $2 / 3$ dos óbitos terem sido diagnosticados durante a internação em que ocorreu o óbito caracterizam diagnóstico tardio e sinalizam a existência de importantes falhas na assistência. Além disso, esses casos contribuem para perpetuação da transmissão massiva dentro das UPs, favorecida pela superlotação das celas e pelas más condições de ventilação. Pode-se ainda esperar alta mortalidade por TB em prisões com alta prevalência de TB e de infecção pelo HIV/aids, como em países africanos 24 ou em países da Europa do Leste 25, onde as formas resistentes ou multirresistentes da TB são especialmente frequentes.

O impacto do HIV/aids na mortalidade prisional, quatro vezes superior entre PPL quando comparadas à população geral do Estado de Rio de Janeiro, poderia ter sido evitado, em particular, com oferta sistemática de teste rápido para HIV, especialmente no momento do ingresso na prisão, o estabelecimento oportuno do tratamento antirretroviral e da profilaxia de infecções oportunistas. A mortalidade por HIV/aids pode apresentar importante variação geográfica no Brasil de acordo com 
as diferenças de prevalência de infecção pelo HIV/aids observadas nas prisões de diferentes regiões do país, relativamente baixa na Região Centro-oeste 26 e particularmente alta no Sul 7 . Entre os óbitos por septicemia, aqueles decorrentes de infecções de pele, poderiam ser evitados pelo maior acesso à água, a material de higiene e a local adequado para lavagem e secagem das roupas 2, além de tratamento precoce e adequado dessas infecções facilmente curáveis.

A evolução desfavorável de doenças crônicas, como a diabetes mellitus (Capítulo IV da CID-10), doenças cardíacas e hipertensão arterial (Capítulo IX da CID-10), observada em nosso estudo, cuja mortalidade foi, entre as PPL, o dobro da observada para a população geral, em parte, pode ser decorrente do modelo inadequado de atenção básica nas UPs, com serviços pouco resolutivos voltados para o atendimento por demanda espontânea, sem protocolos de acompanhamento individualizado dos pacientes nem ações programadas de prevenção de complicações de doenças crônicas. O caso do paciente de 29 anos, diabético insulinodependente, que, durante um período de dois anos, foi atendido, várias vezes, em caráter de urgência, por episódios de descompensação que variavam de hipoglicemia a cetoacidose, e que evoluiu com cegueira, insuficiência renal crônica e quadro de septicemia, que o levou à morte, é emblemático dessa situação.

As mortes violentas entre PPL no Brasil são conhecidas essencialmente pelos episódios de rebelião ou briga entre grupos de presos relatados pela imprensa, com número, muitas vezes, expressivo de mortes em curto espaço de tempo como ocorreu, por exemplo, no Rio de Janeiro (junho de 2004, 34 mortes), no Amazonas (janeiro de 2017, 59 mortes) e em Roraima e no Rio Grande do Norte (janeiro de 2017, 33 e 26 mortes, respectivamente) 18. No entanto, tais eventos, que contribuem para uma imagem da prisão como lugar de extrema violência, não foram observados no período estudado no Rio de Janeiro, e a mortalidade por causa externa foi duas vezes superior na população geral comparada às PPL (SMR = 0,32; IC95\%: 0,24,4-0,40). A violência física interpessoal cotidiana, em geral naturalizada, pode ter sido a causa de parte dos $35 \%$ dos óbitos por causa externa classificados como acidente ou intenção indeterminada. Isso é preocupante e demostra, mais uma vez, a necessidade da realização sistemática de exame de necrópsia em pessoas que falecem durante o encarceramento. Mesmo com menor proporção de óbitos decorrentes de violência física em relação à morte por causas naturais, a prisão não é um espaço de proteção, pois permanece como um espaço onde a alta mortalidade por doenças infecciosas negligenciadas e potencialmente curáveis se insere em um continuum de violência estrutural que, de acordo com a OMS 27, podem ser categorizadas como mortes decorrentes de violência coletiva social por privação ou negligência em relação ao direito e à assistência à saúde 20 .

Diferentemente do observado nas prisões de países de alta renda 28,29, o suicídio foi pouco frequente em nosso estudo, mas sua frequência pode estar subestimada. Chama a atenção o alto percentual de mortes por causas externas classificadas como acidente ou intenção indeterminada, que podem corresponder a suicídio ou agressões, traduzindo um elevado nível de violência intramuros e que, novamente, demonstram a necessidade de investigações rigorosas da causa da morte, inclusive com a realização de necrópsias. A ocorrência de mortes por agressão a pacientes com distúrbios mentais durante crises aponta para a necessidade de rever a atenção ao paciente preso portador de transtorno mental e a prática de deixá-los nas celas superlotadas das prisões, sem o necessário tratamento e acompanhamento psiquiátrico e psicológico que garantam sua proteção.

O fato de $1 / 3$ do total de óbitos ter ocorrido na própria unidade prisional, sem assistência médica, traduz a dificuldade das PPL em acessar o ambulatório da unidade prisional, a incapacidade desses ambulatórios em detectar evoluções desfavoráveis e a dificuldade de acesso a estruturas de saúde intramuros de maior complexidade. Nessa mesma direção, o baixíssimo percentual $(0,7 \%)$ de óbitos ocorridos em hospitais públicos extramuros demostra a falta de acesso da população encarcerada a essas estruturas, o que contradiz os preceitos constitucionais e legais 13,30 e representa um grave desrespeito aos direitos das PPL, que deveriam ter seu acesso garantido a serviços com a mesma qualidade dos oferecidos à população geral ${ }^{31}$. A nosso ver, parecem contribuir para essa situação as dificuldades e certa resistência da administração penitenciária para o transporte e manutenção de escolta em hospitais extramuros e a reticência dos serviços extramuros em receber esses pacientes em decorrência de questões relacionadas à segurança, mas também, muitas vezes, embasadas em ideias preconcebidas sobre as pessoas presas.

O achado em nosso estudo, de taxa global de mortalidade superior na população prisional em relação à população geral do estado, está em contraste com observações de Clavel et al. ${ }^{9}$, Salive et 
al. 10 e Fazel \& Benning 11, em países de alta renda. Essa diferença é essencialmente ligada ao peso muito grande entre as causas de morte nas prisões do Rio de Janeiro de TB que, como mostrado pelo estudo de epidemiologia molecular realizado nas prisões do Rio de Janeiro, é, na maioria dos casos, adquirida na prisão 32 .

A força do presente estudo comparado a estudos anteriormente publicados está nas investigações adicionais que fizemos a partir da coleta de informações nos prontuários dos falecidos e em laudos de necrópsia, o que permitiu a reclassificação da causa mortis em muitos casos.

Entretanto, apresenta limitações, como a maioria dos estudos retrospectivos, especialmente relacionadas às deficiências nos registros de saúde e à impossibilidade de acessar alguns documentos, apesar do grande esforço na busca de informações complementares em diferentes fontes. A proporção de perdas (não localizados no SIM e DO não encontrada) foi relativamente pequena, mas não é possível determinar se concentravam-se em subgrupos específicos. O trabalho meticuloso de revisão das causas de óbito atenuou as limitações dos registros da SEAP-RJ, e a magnitude dos SMR para as causas específicas de óbitos mais frequentes parece representar aproximações razoáveis do sobrerrisco das PPL em relação à população geral. Além disso, a ausência de informações sobre o turnover da população prisional pode ter resultado em superestimação das taxas de mortalidade, e a indisponibilidade de dados sobre o nível socioeconômico da população geral no SIM impossibilitou considerar essa variável na padronização.

\section{Conclusão}

A importante mortalidade por doenças que observamos, com expressiva contribuição das doenças infecciosas, em sua maioria evitáveis, uma vez que são de fácil diagnóstico e têm tratamento disponível na rede pública, traduz grave desassistência. Isso implica na elaboração e implementação de uma verdadeira política de saúde pública nas prisões do Estado do Rio de Janeiro que permita efetivamente integrar as PPL ao SUS, a fim de que possam se beneficiar da mesma atenção à saúde que a população geral, por meio da implementação da PNAISP, que ainda é muito incipiente nas prisões do estado 33 . No contexto atual de COVID-19, avaliações da mortalidade são especialmente importantes, entre outros indicadores, para avaliar a reposta do sistema de saúde prisional à pandemia.

Nosso estudo mostra a pertinência da utilização da mortalidade como indicador do acesso e da resolutividade dos serviços de saúde para as PPL, contribuindo para a readequação das ações de promoção da saúde, prevenção e assistência, o que torna necessário o desenvolvimento de um sistema de monitoramento em tempo real dos óbitos entre os PPL. É também prioritária a realização, com objetivos operacionais, de pesquisas avaliativas sobre a estruturação e o funcionamento do sistema de saúde prisional e as modalidades de implementação da PNAISP.

Entre outros indicadores, a mortalidade, indicador especialmente sensível para avaliar o acesso, a qualidade e a resolutividade do serviço, permitirá dar maior visibilidade, aos gestores e à sociedade, das condições de vida e de acesso dessa população aos serviços de saúde que, no Brasil, são um direito constitucional 12, possibilitando dimensionar o grau de exclusão das PPL do acesso ao SUS 30. Mas, como ressaltam Le Marcis \& Attas 34 (p. 584), "a ilegitimidade social da população encarcerada e a reticência dos Estados em propor aos condenados o que eles não garantem ao conjunto da população são dois argumentos colocados em evidencia para justificar o que se assemelha a uma necropolítica, uma política do abandono, da negligência e da morte associada ao encarceramento".

A pertinência e a adequação da reflexão, a partir da realidade retratada neste estudo, tornam ainda mais premente a criação de ferramentas e mecanismos independentes de fiscalização das ações de prevenção e assistência à saúde nas prisões, visando assegurar que o direito à saúde e à vida das PPL sejam respeitados. 


\section{Colaboradores}

A. Sánchez contribuiu na concepção e planejamento do estudo, análise e interpretação dos dados, revisão crítica do conteúdo, redação do manuscrito e aprovação da versão final. C. R. S. Toledo contribuiu na concepção e planejamento do estudo, análise dos dados, elaboração do manuscrito e revisão da versão final. L. A. B. Camacho contribuiu na definição do desenho do estudo, análise, elaboração e revisão final do manuscrito. B. Larouze contribuiu na concepção e planejamento do estudo, análise dos dados, elaboração do manuscrito e revisão final.

\section{Informações adicionais}

ORCID: Alexandra Sánchez (0000-0001-56171173); Celina Roma Sánchez de Toledo (00000003-1121-8411); Luiz Antônio Bastos Camacho (0000-0003-4656-1914); Bernard Larouze (00000001-9906-6293).

\section{Agradecimentos}

Agradecemos à Secretaria de Estado de Administração Penitenciária (SEAP-RJ), à Secretaria de Estado de Saúde do Rio de Janeiro (SES-RJ) e ao Ministério Público do Rio de Janeiro (MPRJ), que tornaram este estudo possível. Este estudo foi financiado pelo Programa INOVA Fiocruz (Conhecimento) da Fundação Oswaldo Cruz e CNPq Universal do Conselho Nacional de Desenvolvimento Científico e Tecnológico.

\section{Referências}

1. Ministério da Justiça. Levantamento Nacional de Informações Penitenciárias. Sistema Penitenciário no Brasil, dados consolidados, junho 2017. http://depen. gov.br/DEPEN/depen/sisdepen/infopen/ relatorios-sinteticos/infopen-jun-2017rev-12072019-0721.pdf (acessado em 10/ $\mathrm{Jul} / 2020)$.

2. Ferreira AC, Sereno GC, Fernandes IS, Dias JM, Ferreira NDP, Albergaria RCB. Relatório parcial sobre os impactos do COVID-19 no Sistema Penitenciário do Rio de Janeiro. Informações adicionais até 5 de julho de 2020. http:// mecanismorj.com.br/wp-content/uploads/ Relat\%C3\%B3rio-parcial-do-MEPCTRJsobre-o-COVID19-no-sistema-prisional-atu alizado-05.07.pdf (acessado em 10/Jul/2020).

3. Job Neto F, Miranda RB, Coelho RA, Gonçalves CP, Zandonade E, Miranda AE. Health morbidity in Brazilian prisons: a time trends study from national databases. BMJ Open 2019; 9:e026853.

4. Sánchez A, Massari V, Gerhardt G, Espinola AB, Siriwardana M, Camacho LAB, et al. X ray screening at entry and systematic screening for the control of tuberculosis in a highly endemic prison, Rio de Janeiro. BMC Public Health 2013; 13:983-90.

5. Bourdillon PM, Gonçalves CC, Pelissari DM, Arakaki-Sanchez D, Ko AI, Croda J, et al. Increase in tuberculosis cases among prisoners, Brazil, 2009-2014. Emerg Infect Dis 2017; 23:496-9.

6. El Maerrawi I, Carvalho HB. Prevalence and risk factors associated with HIV infection, hepatitis and syphilis in a state prison of São Paulo. Int J STD AIDS 2015; 26:120-7.

7. Prellwitz M, Alves B, Ikeda ML, Kuhleis D, Picon P, Jarczewski C, et al. HIV behind bars: human immunodeficiency virus 1 cluster analysis and drug resistance in a reference correctional unit from southern Brazil. PLoS One 2013; 8:e69033. 
8. Brasil. Política Nacional de Atenção Integral à Saúde das Pessoas Privadas de Liberdade no Sistema Prisional. http://bvsms. saude.gov.br/bvs/saudelegis/gm/2014/ pri0001_02_01_2014.html (acessado em 10/ $\mathrm{Jul} / 2018$ ).

9. Clavel F, Benhamou S, Flamant R. Decreased mortality among male prisoners. Lancet 1987; 2:1012-4.

10. Salive ME, Smith GS, Brewer TF. Death in prison: changing mortality patterns among male prisoners in Maryland, 1979-87. Am J Public Health 1990; 80:1479-80.

11. Fazel S, Benning R. Natural deaths in male prisoners: a 20-year mortality study. Eur J Public Health 2006; 16:441-4.

12. Patterson EJ. Incarcerating death: mortality in U.S. state correctional facilities, 1985-1998. Demography 2010; 47:587-607.

13. Brasil. Constituição da República Federativa do Brasil de 1988. http://www.planalto.gov. br/ccivil_03/constituicao/constituicao.htm (acessado em 16/jul/2020).

14. Costa F, Bianchi P. "Massacre silencioso": doenças tratáveis matam mais que violência nas prisões brasileiras. UOL Notícias 2017; 14 ago. https://noticias.uol.com.br/cotidiano/ultimasnoticias/2017/08/14/massacre-silenciosomortes-por-doencas-trataveis-superam-mor tes-violentas-nas-prisoes-brasileiras.htm.

15. Secretaria de Estado de Administração Penitenciária do Rio de Janeiro. Resolução SEAP no 507, de 21 de novembro de 2013. Estabelece rotina administrativa para casos de óbitos internos ocorridos no âmbito das unidades prisionais e hospitalares da Secretaria de Estado de Administração Penitenciária - SEAP. Diário Oficial do Estado do Rio de Janeiro 2013; 22 nov.

16. Szklo M, Nieto FJ. Epidemiology beyond the basics. 4th Ed. Burlington: Jones \& Bartlett Learning; 2019.

17. Abramson JH. WINPEPI updated: computer programs for epidemiologists, and their teaching potential. Epidemiol Perspect Innov 2011; $8: 1$.

18. Supremo Tribunal Federal. Tema 365: responsabilidade do Estado por danos morais decorrentes de superlotação carcerária. RE 580252. http://portal.stf.jus.br/processos/detalhe. asp? incidente $=2600961$.

19. Le Marcis F. A impossível governança da saúde em prisão? Reflexões a partir da MACA (Costa do Marfim). Ciênc Saúde Colet 2016; 21:2011-20.

20. Chies LAB, Almeida B. Mortes sub custódia prisional no Brasil. Prisões que matam; mortes que pouco importam. Revista de Ciencias Sociales $2019 ; 32: 67-90$.

21. Diuana V, Lhuilier D, Sánchez AR, Amado G, Araújo L, Duarte AM, et al. Saúde em prisões: representações e práticas dos agentes de segurança penitenciária no Rio de Janeiro, Brasil. Cad Saúde Pública 2008; 24:1887-96.
22. Rosen DL, Wohl DA, Schoenbach VJ. All-cause and cause-specific mortality among black and white North Carolina state prisoners, 19952005. Ann Epidemiol 2011; 21:719-26.

23. Secretaria de Vigilância em Saúde, Ministério da Saúde. Tuberculose 2020. Boletim Epidemiológico 2020; Número Especial. https:// www.saude.gov.br/images/pdf/2020/mar co/24/Boletim-tuberculose-2020-marcas--1-. pdf.

24. Telisinghe L, Charalambous S, Topp SM, Herce ME, Hoffmann CJ, Barron P, et al. HIV and tuberculosis in prisons in sub-Saharan Africa. Lancet 2016; 388:1215-27.

25. Altice FL, Azbel L, Stone J, Brooks-Pollock E, Smyrnov P, Dvoriak S, et al. The perfect storm: incarceration and the high-risk environment perpetuating transmission of HIV, hepatitis C virus, and tuberculosis in Eastern Europe and Central Asia. Lancet 2016; 388:1228-48.

26. Sgarbi RVE, Carbone ASS, Paião DSG, Lemos EF, Simionatto S, Puga MAM, et al. A Crosssectional survey of HIV testing and prevalence in twelve Brazilian correctional facilities. PLoS One 2015; 10:e0139487.

27. Organização Mundial da Saúde. Relatório mundial sobre violência e saúde. https://opas. org.br/wp-content/uploads/2015/09/relato rio-mundial-violencia-saude.pdf (acessado em 16/Jul/2020).

28. Fazel S, Benning R, Danesh J. Suicides in male prisoners in England and Wales, 1978-2003. Lancet 2005; 366:1301-2.

29. Radeloff D, Lempp T, Kettner M, Rauf A, Bennefeld-Kersten K, Freitag CM. Male suicide rates in German prisons and the role of citizenship. PLoS One 2017; 12:e0178959.

30. Brasil. Lei no 8.080 de 19 de setembro de 1990. Dispõe sobre as condições para a promoção, proteção e recuperação da saúde, organização e funcionamento dos serviços correspondentes e dá outras providências. Diário Oficial da União 1990; 20 set.

31. Larouzé B, Ventura M, Sánchez AR, Diuana V. Tuberculose nos presídios brasileiros: entre a responsabilização estatal e a dupla penalização dos detentos. Cad Saúde Pública 2015; 31:1127-30.

32. Sánchez A, Huber FD, Massari V, Barreto A, Camacho LAB, Cesconi V, et al. Extensive $M y$ cobacterium tuberculosis circulation in a highly endemic prison. Need for urgent environmental interventions. Epidemiol Infect 2012; 140:1853-61.

33. Simas L, Sánchez A, Ventura M, Diuana V, Larouze B. Análise crítica do modelo de atenção à saúde das pessoas privadas de liberdade no Brasil. Cad Ibero Am Direito Sanit 2021; 10:39-55.

34. Le Marcis F, Attas F. Epidemics and COVID-19 in African prisons: towards an inclusive approach of health. Sante Publique 2020; 32: 583-7. 


\section{Abstract}

Mortality in prisons, a basic indicator of the right to health for incarcerated persons, has never been studied extensively in Brazil. An assessment of allcause and cause-specific mortality in prison inmates was conducted in 2016-2017 in the state of Rio de Janeiro, based on data from the Mortality Information System and Prison Administration. Mortality rates were compared between prison population and general population after standardization. The leading causes of death in inmates were infectious diseases (30\%), cardiovascular diseases (22\%), and external causes (12\%). Infectious causes featured HIV/AIDS (43\%) and TB (52\%, considering all deaths with mention of TB). Only $0.7 \%$ of inmates who died had access to extramural health services. All-cause mortality rate was higher among prison inmates than in the state's general population. Among inmates, mortality from infectious diseases was 5 times higher, from TB 15 times higher, and from endocrine diseases (especially diabetes) and cardiovascular diseases 1.5 and 1.3 times higher, respectively, while deaths from external causes were less frequent in prison inmates. The study revealed important potentially avoidable excess deaths in prisons, reflecting lack of care and exclusion of this population from the Brazilian Unified National Health System. This further highlights the need for a precise and sustainable real-time monitoring system for deaths, in addition to restructuring of the prison staff through implementation of the Brazilian National Policy for Comprehensive Healthcare for Persons Deprived of Freedom in the Prison System in order for inmates to fully access their constitutional right to health with the same quality and timeliness as the general population.

Prisons; Mortality; Cause of Death; Tuberculosis; Epidemiological Monitoring

\section{Resumen}

La mortalidad en las prisiones, indicador fundamental del derecho a la salud de personas privadas de libertad (PPL), nunca se estudió profundamente en Brasil. La evaluación de la mortalidad global y por causas entre PPL, encarceladas en 2016-2017 en el estado de Rio de Janeiro, se realizó a partir de datos del Sistema de Información de Mortalidad y la Administración Penitenciaria. Se compararon las tasas de mortalidad entre PPL y población general del estado tras la estandarización. Las principales causas de muerte entre PPL fueron: enfermedades infecciosas (30\%), enfermedades del aparato circulatorio (22\%) y causas externas (12\%). Entre las causas infecciosas, se destacan VIH/sida (43\%) y tuberculosis (TB) (52\%, si se consideran todos los óbitos con mención TB). Solamente un 0,7\% de las PPL que fallecieron tuvieron acceso a un servicio de salud extramuros. La tasa global de mortalidad fue mayor entre las PPL, comparada con la población general del estado. Entre las PPL, la mortalidad por enfermedades infecciosas fue 5 veces superior, por TB 15 veces, $y$ por enfermedades endocrinas, especialmente diabetes, $y$ enfermedades circulatorias $(1,5$ y 1,3 veces respectivamente), mientras que las muertes por causa externa fueron menos frecuentes entre PPLs. Este estudio muestra un expresivo exceso de muertes potencialmente evitables en las prisiones, lo que se traduce en una importante desasistencia y la exclusión de esta población del Sistema Único de Salud. Pone en evidencia la necesidad de un sistema de supervisión en tiempo real de los óbitos, preciso y sostenible, además de la reestructuración de la salud en las prisiones, mediante la efectivización de la Politica Nacional de Atención Integral a la Salud de las Personas Privadas de Libertad en el Sistema Penitenciario para que las PPLs disfruten del derecho constitucional a la salud en su integridad, con la misma calidad y oportunidad ofrecida a la población general.

Prisiones; Mortalidad; Causas de Muerte; Tuberculosis; Monitoreo Epidemiológico

Recebido em 14/Set/2020

Versão final reapresentada em 21/Jan/2021

Aprovado em 26/Jan/2021 